\title{
Surgical-site Infection following Cesarean Section in Haiti: Incidence and Associated Pathogens

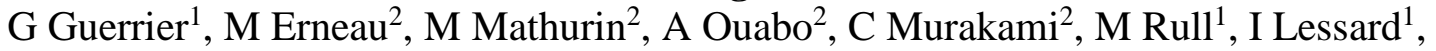

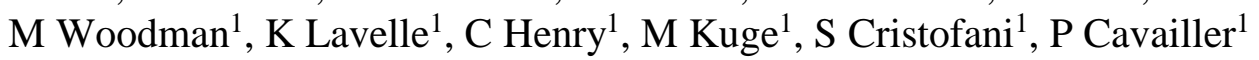

\begin{abstract}
Background: Surgical site infection (SSI) after a Cesarean section (CS) increases maternal morbidity and medical costs. No data regarding the epidemiology of SSI after CS are available in settings operated by Médecins Sans Frontières.

Objectives: To determine incidence and independent risk factors for SSI after CS, as well as pathogens associated with infections.

Methods: A prospective data collection among women undergoing CS was conducted in a 43-bed maternity ward hospital in Haiti. Infections were identified during hospital stay or by post-discharge survey using a combination of telephone calls, healthcare worker questionnaires and outpatient medical records review for 30 days after surgery. Surgical site infection was defined according to the International Nosocomial Infection Surveillance system criteria.

Results: From $1^{\text {st }}$ of May to $30^{\text {th }}$ of November 2014,523 women were included in the study. Eight SSI were identified, yielding an SSI rate of 1.5 infections/100 (95\% CI 0.5-2.6). All of them were detected during post-discharge surveillance. Mean hospital stay was four days, while mean time between CS and SSI diagnosis was 12 days. Lost to follow-up rates were 39\%, 57\%, 89\% after seven days, 15 days, and 30 days, respectively. The most common pathogen isolated was methicillin-resistant Staphylococcus aureus (MRSA).
\end{abstract}

Conclusions: Post-discharge surveillance system is crucial to detect and treat SSI. Efforts should be made to optimize post-operative follow-up and measures should be taken to mitigate the spread of MRSA.

From: ${ }^{1}$ Médecins Sans Frontières, Geneva, Switzerland and ${ }^{2}$ Médecins Sans Frontières, Leogane, Haiti.

Correspondence: Dr G Guerrier, Médecins Sans Frontières, Geneva, Switzerland.

E-mail: guerriergilles@gmail.com

Conflict of interest: none declared

The authors declare no conflict of interest/funding sources external to MSF. 


\section{INTRODUCTION}

Post-operative surgical site infections (SSI) rate is considered a key indicator of the quality of surgical and post-operative care performed by hospitals (1). The occurrence of SSI is associated with an increased morbidity, mortality and costs of healthcare (2). The routine use of surveillance systems for infection and reports of SSI rates to surgeons have contributed significantly to the reduction of the incidence of SSI and to the improvement of patient care $(3,4)$.

However, the magnitude of the problem is probably underestimated, since $19 \%$ to $65 \%$ of SSIs are first diagnosed only after a patient's discharge from the hospital (5-7), including in obstetrics and gynaecology patients $(8,9)$. To be accurate, the surveillance of SSI should be performed not only in-hospital but also outside the traditional hospital setting in order to clearly estimate the real incidence of post-operative SSI (10). With the increased financial pressure to shorten post-operative stays and to perform surgical procedures in outpatient clinics, it may be expected that an increasing number of SSIs will be detected only after hospital discharge.

Several surveillance methods have been proposed to detect post-discharge SSI. These include routine direct wound examination, out-patient chart review by a trained professional, surgeon-reporting by mail or by survey, patient reporting by mail or telephone and microbiology data $(4,8,11)$. However, a systematic review of the methods used to identify SSI following discharge from hospital concluded that existing studies on the subject have so far failed to identify a valid, reliable method for identifying such infections during the post discharge period. On a local level, the method used to identify post discharge SSI is likely to depend on existing resources, on the objective of surveillance and on the nature of the data routinely available (12). Due to human, logistical or environmental constraints, such costly and time-consuming efficient methods pose a real challenge in resource-limited settings (13). 
Information on strategies matching available resources in developing countries is scarce (14). Moreover, to our knowledge, there is no published data on the effectiveness of SSI surveillance in programmes run by non-governmental organizations such as Médecins Sans Frontières (MSF). The purpose of this study was to conduct a combined in-hospital and postdischarge surveillance in obstetric patients, in order to identify cases of SSI and to document associated pathogens in a Haitian hospital run by MSF.

\section{MATERIAL AND METHODS}

The study was carried out in a 43-bed maternity ward hospital run by MSF, located in Leogane (population around 200 000), Haiti. The hospital had an active infection control committee and program before the start of the study. From $1^{\text {st }}$ of May to $30^{\text {th }}$ November 2014 , all patients attending the obstetrics department who underwent a Cesarean section were enrolled in an observational study for the detection of SSIs.

In-hospital SSI was diagnosed by a daily review of data gathered by the hospital's infection control committee. Information recorded included results of microbiology testing, chart reviewing for antibiotic prescriptions and nurse's notes and obstetrical ward rounds surveillance as indicators of SSI in Cesarean section patients.

In addition to in-hospital surveillance, all patients undergoing Cesarean section were instructed to return to the outpatient clinic by the $10^{\text {th }}$ to the $15^{\text {th }}$ post-operative day. A phone call follow-up was scheduled between day 20 and day 30 after discharge. In this clinic, members of the nosocomial infection committee removed the stitches and assessed the surgical wound for the existence of SSI. At the time of the clinic visit, patients were required to come back for a new assessment if signs and symptoms of infection were noted. 
A case definition of SSI included infection which had occurred within 30 days after surgical procedure and had restricted to skin and subcutaneous tissue, in addition to at least one of the following criteria:

1. Purulent drainage from the incision;

2. Organism isolated from an aseptically obtained culture of fluid or tissue from the incision; and

3. At least one of the following signs or symptoms of infections at the surgical site: pain, tenderness, swelling, redness or heat.

As a routine, all patients undergoing Cesarean section received prophylactic antibiotic therapy (Cephazolin $1.0 \mathrm{~g}$ given intravenously just after cord clamping).

\section{RESULTS}

A total of 1605 deliveries were performed during the study period, $1082(67 \%)$ by the vaginal route and $523(33 \%)$ by Cesarean section. In-hospital surveillance was performed on all patients. Post-discharge examinations were done on $523(100 \%)$ of the 523 Cesarean section patients. A total of $319(61 \%)$ patients returned to the outpatient clinic for post-discharge surveillance during the first week after discharge. The incidence of SSI in Cesarean section detected by in-hospital surveillance was $1.5 \%$ ( 8 cases). All of them were detected during post-discharge period. Median hospital stay was four days while median elapsed time between CS and SSI diagnosis was 12 days. The proportions of lost to follow-up rates were 39\%, 57\%, 89\% after seven days, 15 days, and 30 days, respectively. All SSIs detected were restricted to superficial sites. Ninety-five per cent of the infections were detected between the $10^{\text {th }}$ and $15^{\text {th }}$ day after hospital discharge. The most common pathogen isolated was methicillin-resistant Staphylococcus aureus (MRSA) [4 cases]. Other isolated pathogens 
included Escherichia coli, Klebsiella pneumoniae, Enterococcus faecalis, Acinetobacter baumanii, Morganella morganii and Pseudomonas aeruginosa. Multiple pathogen infections were found in four cases.

\section{DISCUSSION}

The results of our investigation clearly highlight the important differences between inhospital and post-discharge surveillance for detecting SSI in the obstetric population. The high incidence of SSIs following Cesarean section after discharge reported also in other studies indicates that failing to conduct follow-up evaluation of these patients necessarily results in a substantial miscalculation of the real SSI rate. Compared to a global estimation of SSI in resource-limited settings (15). There is little doubt that SSI rates are under-estimated in our study. Some infected patients might not have returned for follow-up due to socioeconomic limitation; finally some of them might have seek care to private physician's sector.

However, we believe high quality of nursing care contributed to the low SSI rate reported. In our study population, most of the SSIs were detected between the $10^{\text {th }}$ and $15^{\text {th }}$ days after hospital discharge. Therefore, the 30-day period of surveillance for SSIs suggested by CDC appears to be more than is necessary for obstetrical patients.

Our findings confirm the need to perform some type of post-discharge surveillance to detect SSIs. However, the best way to conduct the post-discharge surveillance in resourcelimited countries is not clearly established. The ideal methodology should not be timeconsuming and should be cost-effective and have high sensitivity and specificity. Using follow-up cards and mobile phone technology, the purulent discharge criterion to diagnose non-obstetrical SSI after discharge yielded a sensitivity of $83 \%$ and a specificity of $100 \%$ in a Cambodian hospital (14). Although no consensus has yet been reached on the best 
methodology for implementing post-discharge surveillance, telephone contact would appear to represent a low-cost technique that requires minimal resources. Nevertheless, some studies reported a low positive predictive value (30\%) for diagnoses made according to patient telephone reports, although the negative predictive value was high (98\%) compared with diagnosis made by an infection control nurse through direct examination of the surgical incision in the patient following discharge from hospital (16).

In a challenging context with limited resources, physicians in this Haitian hospital strongly adhere to the current MSF guidelines recommending/prescribing systematic shortcourse narrow-spectrum prophylactic antibiotics before skin incision. We found an alarmingly high proportion of methicillin-resistant Staphylococcus aureus (MRSA). All MRSA isolates were resistant to ceftriaxone, erythromycin, gentamicin and penicillin but were $100 \%$ sensitive to vancomycin, rifampin and chloramphenicol. Although the importance of MRSA in Haiti is not well documented, some studies showed a sharp increase in MRSA prevalence over time in other Caribbean countries, such as Trinidad (17), Martinique, and Dominican Republic (18). Fortunately, most SSIs detected in our patients were restricted to superficial sites and were not related to adverse outcomes, increases in length of hospitalization, or required vancomycin administration. However, more severe surgical site infections could be expected to occur as SSI rates increase with resistant pathogens. For this reason, surveillance cultures of both patients and healthcare workers may help to identify carriers who would be offered antibiotics to eradicate the organisms. Most MRSA are resistant to several non- $\beta$-lactam antibiotics. Infection control measures aimed at the proper hand hygiene procedures may interrupt the spread of MRSA. Frequent monitoring of susceptibility patterns of MRSA and the formulation of a definite antibiotic policy may be helpful in decreasing the incidence of MRSA infection. The continuing surveillance of 
population-specific infection rates and reporting of them to practitioners could allow prompt intervention measures if unexpected outbreaks occurred.

As the hospital program was about to close the study duration was restricted to seven months and the expected sample size of our study was not sufficient to explore the potential risk factors for SSI following C-section.

\section{CONCLUSION}

Surgical site infection surveillance in such resource-limited settings proved to be challenging. Adequate, culturally acceptable, and innovative procedures after discharge to assess the wound healing process of patients should be implemented to optimize post-discharge followup of patients after C-section. Post discharge follow-up rate may be increased by reimbursement of transportation fees. In addition to surgical complication follow-up, a postsurgery visit scheduled at day 15 could be combined with a post-natal visit, which could provide the mothers with obstetric follow-up, contraceptive counselling and newborn care. Post-surgery follow-up should also be routinely performed for other types of surgical procedures in Haiti, in accordance with the National Infection Control Programme. 


\section{REFERENCES}

1. Gaynes RP. Surveillance of nosocomial infections: a fundamental ingredient for quality. Infect Control Hosp Epidemiol 1997; 18: 475-8.

2. Roy MC, Perl TM. Basics of surgical-site infection surveillance. Infect Control Hosp Epidemiol 1997; 18: 659-68.

3. Emmerson AM, Enstone JE, Kelsey MC. The Second National Prevalence Survey of infection in hospitals: methodology. J Hosp Infect 1995; 30: 7-29.

4. Pottinger JM, Herwaldt LA, Perl TM. Basics of surveillance-an overview. Infect Control Hosp Epidemiol 1997; 18: 513-27.

5. Holtz TH, Wenzel RP. Postdischarge surveillance for nosocomial wound infection: a brief review and commentary. Am J Infect Control 1992; 20: 206-13.

6. Medina-Cuadros M, Sillero-Arenas M, Martínez-Gallego G, Delgado-Rodríguez M. Surgical wound infections diagnosed after discharge from hospital: epidemiologic differences with in-hospital infections. Am J Infect Control 1996; 24: 421-8.

7. Sands K, Vineyard G, Platt R. Surgical site infections occurring after hospital discharge. J Infect Dis 1996; 173: 963-70.

8. Baker C, Luce J, Chenoweth C, Friedman C. Comparison of case-finding methodologies for endometritis after cesarean section. Am J Infect Control 1995; 23: $27-33$.

9. Gravel-Tropper D, Oxley C, Memish Z, Garber GE. Underestimation of surgical site infection rates in obstetrics and gynecology. Am J Infect Control 1995; 23: 22-6.

10. Ferraz EM, Ferraz AA, Coelho HS, Pereira Viana VP, Sobral SM, Vasconcelos MD et al. Postdischarge surveillance for nosocomial wound infection: does judicious monitoring find cases? Am J Infect Control 1997; 25: 102-5. 
11. Manian FA. Surveillance of surgical site infections in alternative settings: exploring the current options. Am J Infect Control 1995; 23: 290-4.

12. Petherick ES, Dalton JE, Moore PJ, Cullum N. Methods for identifying surgical wound infection after discharge from hospital: a systematic review. BMC Infect Dis 2006; 6: 170.

13. Allegranzi B, Bagheri Nejad S, Combescure C, Graafmans W, Attar H, Donaldson L et al. Burden of endemic health-care-associated infection in developing countries: systematic review and meta-analysis. Lancet 2011; 377: 228-41.

14. Guerra J, Guichon C, Isnard M, So S, Chan S, Couraud S et al. Active prospective surveillance study with post-discharge surveillance of surgical site infections in Cambodia. J Infect Public Health 2015; 8: 298-301.

15. Nyamogoba H, Obala AA. Nosocomial infections in developing countries: cost effective control and prevention. East Afr Med J 2002; 79: 435-441. Review.

16. Whitby M, McLaws ML, Collopy B, Looke DF, Doidge S, Henderson B et al. Postdischarge surveillance: can patients reliably diagnose surgical wound infections? J Hosp Infect 2002; 52: 155-160.

17. Orrett FA, Land M. Methicillin-resistant Staphylococcus aureus prevalence: current susceptibility patterns in Trinidad. BMC Infect Dis 2006; 6: 83.

18. Uhlemann AC, Dumortier C, Hafer C, Taylor BS, Sánchez EJ, Rodriguez-Taveras C et al. Molecular characterization of Staphylococcus aureus from outpatients in the Caribbean reveals the presence of pandemic clones. Eur J Clin Microbiol Infect Dis 2012; 31: 505-511. 